\title{
The detection of the rich p-mode spectrum and asteroseismology of Przybylski's star ${ }^{\star}$
}

\author{
D. E. Mkrtichian ${ }^{1,2}$, A. P. Hatzes ${ }^{3}$, H. Saio ${ }^{4}$, and R. R. Shobbrook ${ }^{5}$
}

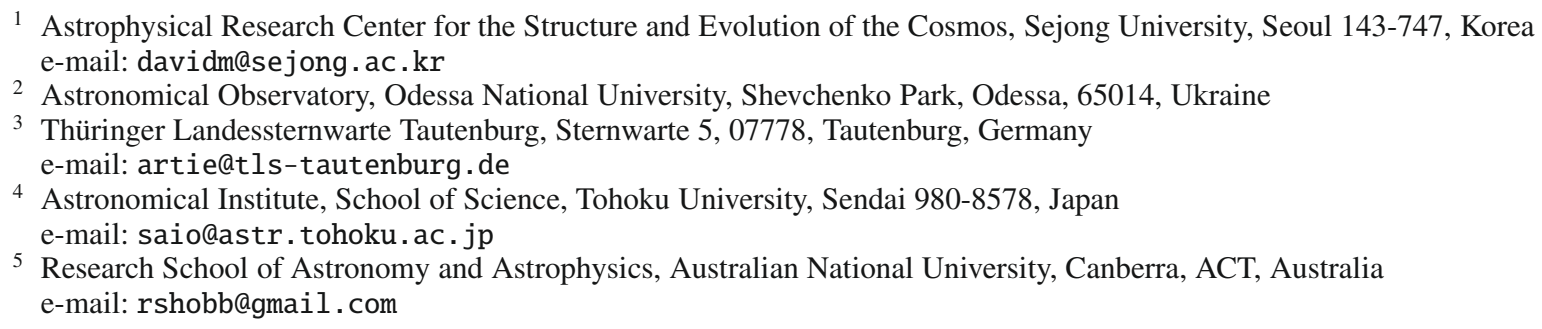

Received 2 April 2008 / Accepted 23 August 2008

\section{ABSTRACT}

\begin{abstract}
Aims. We investigate the oscillation spectrum of the most chemically-peculiar star in the sky, the rapidly-oscillating magnetic (roAp) star HD 101065.

Methods. High-precision radial velocity (RV) measurements, spanning four consecutive nights on 3-6 March, 2004, were obtained with the HARPS echelle-spectrometer at the ESO 3.6-m telescope, and photometric data were acquired during 6 nights of observations at the 24-inch telescope of Siding Spring Observatory. The RVs were measured using all spectral lines in the wavelength range 4400-5100 A.

Results. We detected a rich spectrum of oscillation modes with semi-amplitudes ranging between $217 \mathrm{~m} \mathrm{~s}^{-1}$ and $1.6 \mathrm{~m} \mathrm{~s}^{-1}$. These belong to the complete spectrum of high-order, low $\ell=0-2$ modes, having a large spacing of $\overline{\Delta v}=64.07 \pm 0.9 \mu \mathrm{Hz}$. We calculated the nonadiabatic frequencies of axisymmetric high-order p-modes for main-sequence models with dipole magnetic fields of two chemical compositions $(X, Z)=(0.7,0.02)$ and $(X, Z)=(0.695,0.025)$. The former composition provided the best fit model and yielded stellar parameters of mass, $M=1.525 \pm 0.025 M_{\odot}$, age $=(1.5 \pm 0.1) \times 10^{9} \mathrm{yr}$, effective temperature $\log T_{\mathrm{e}}=3.821 \pm 0.006$, luminosity $\log L / L_{\odot}=0.797 \pm 0.026$, surface gravity $\log g=4.06 \pm 0.04$, and polar magnetic field strength, Bp $=8.7 \pm 0.3 \mathrm{kG}$. We are able to identify the modes of all detected oscillation frequencies. The photometric $U$ and $B$ light curves have maxima that precede the RV maximum by $0.16-0.19$ in phase.
\end{abstract}

Key words. stars: chemically peculiar - stars: oscillations - stars: individual: HD 101065

\section{Introduction}

HD 101065 (Przybylski's star) was discovered by Przybylski (1961) as an extremely chemically peculiar star with strong spectral lines of rare-earth elements. The four magnetic field measurements by Wolff \& Hagen (1976) showed a longitudinal magnetic field in the range of $B_{1}=-2100$ to $-2500 \pm 450$ Gauss and this firmly established the magnetic nature of this Ap star. Recent magnetic field measurements by Hubrig et al. (2004) showed half the value of the mean longitudinal magnetic field, $B_{1}=-1014 \pm 72$ Gauss.

In earlier investigations, Wegner \& Petford (1974) identified the spectral lines of 51 chemical elements in HD 101065. Cowley et al. (2000) extended the abundance determination for 54 elements and confirmed the extraordinary +4 to +5 dex chemical overabundance of lantanides. Cowley et al. (2004) claimed the presence of lines of Tc and the short-lived (halflife $t=17.7$ years) element Pm in the spectrum of HD 101065 . The abundance and the isotopic content of $\mathrm{Li}$ was determined by Shavrina et al. (2003, 2006). Gopka et al. (2004) investigated the abundance of the elements $\mathrm{Tc}, \mathrm{Pb}$, and $\mathrm{Bi}$. Remarkably, they also

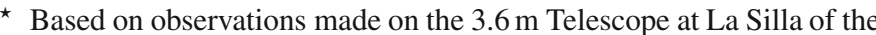
European Southern Observatory under program 072.D-0286.
}

reported the identification of spectral lines of all short-lived radioactive elements with atomic numbers from $Z=84$ to $Z=99$, except for $Z=85$ and 87 (At and Fr). A similar result was reported by Bidelman (2005). Yushchenko et al. (2006) found limits on the abundance of short-lived actinides in the atmosphere of HD 101065. Atmospheric modelling with new transition probabilities for a set of Pm lines (Fivet et al. 2007) and Ra and Ac lines (Quinet et al. 2007) supported the detection of these ions in HD 101065.

The mechanism producing the strong chemical anomalies in Ap stars is still unknown, although the vertical diffusion of elements in the presence of a magnetic field (Michaud 1970) is the one that is largely accepted. This theory predicts the vertical stratification of some elements and their accumulation in the upper atmospheres of Ap stars. However, in view of the recent discoveries of radioactive elements in Ap stars, the diffusion theory alone cannot completely explain the presence of these elements. Goriely (2007) re-examined the role of the spallation process and found that nuclear reactions can explain the full peculiar abundance pattern, including Pm observed in HD 101065, although a highly energetic particle irradiation event is needed.

After the Sun for which the abundances of 73 chemical elements have been determined, HD 101065 is the second star with the most number of identified chemical elements. However, 
in spite of a 44 year-long history of investigations, more than half of the spectral lines in this star are still not identified and the extraordinary chemical anomalies of HD 101065 remain an enigma. Even among the class of peculiar Ap stars HD 101065 qualifies as "the most peculiar star in the sky". The nature of the strong abundance anomalies in HD 101065 as well as the whole class of Ap stars, is one of the great unsolved problems of stellar astrophysics.

The rapid oscillations of HD 101065 were found by Kurtz (1978) and it became a prototype star of a new class of highovertone rapidly-oscillating Ap (roAp) stars exhibiting nonradial acoustic oscillations aligned with the magnetic axis (oblique pulsator). Photometric data on HD 101065 spanning 10 years were studied by Martinez \& Kurtz (1990) who found three periodic signals: the principal frequency $\nu_{1}=1372.89 \mu \mathrm{Hz}$ (with first harmonic at $2 v_{1}=2745.67 \mu \mathrm{Hz}$ ), $v_{2}=1369.63 \mu \mathrm{Hz}$, and $\nu_{3}=1315.07 \mu \mathrm{Hz}$. They concluded that their detected frequencies could not be identified with consecutive overtone modes and tentatively suggested a frequency spacing of $v_{1}$ $v_{3}=57.8 \mu \mathrm{Hz}$. The detailed historical review of HD 101065 investigations and the problems associated with the stellar parameters of star was given by Kurtz \& Martinez (2000). Thirty years after the discovery of pulsations in this star the oscillation spectrum has still not adequately been investigated and the basic stellar parameters remain uncertain.

Recently, Mkrtichian \& Hatzes (2005) presented a preliminary analysis of RVs obtained with the HARPS spectrograph using spectral lines in the sub-interval $\lambda \approx 5200-6000 \AA$. They reported the detection of five individual modes belonging to low $\ell$-degree spectrum of p-modes and having a frequency spacing of about $65 \mu \mathrm{Hz}$. Kurtz et al. (2005) using RV measurements of Pr III lines taken with the VLT found three individual oscillation modes.

The detection of multi-mode oscillations in roAp stars is very important for atmospheric studies in light of the discovery of the short wavelength scale of acoustic oscillations in the atmospheres of Ap stars (Baldry et al. 1998). This opens up new prospects for the study of chemical stratification in the atmosphere of these stars. Mkrtichian et al. (2003) suggested a new, acoustic approach for the study of the chemical profiles in the stellar atmosphere using multimode acoustic cross sections. In this technique the pulsation phases and amplitudes of spectral lines of different ions provide the only true link between spectral lines and the geometric depth of their formation. The multimode oscillations having different acoustic profiles across the line-forming atmosphere is a necessary requirement for a successful application of such a technique.

In this paper we present results of a more detailed radial velocity study of HD 101065 using the HARPS spectroscopy that was initially presented by Mkrtichian \& Hatzes (2005). The aim is to detect and identify the lowest amplitude p-mode oscillations for this star. The supporting photometric observations were carried out to get information about pulsation phase of the photosphere where the continuum is formed. The RV analysis is primarily performed on the "integral" radial velocities representing an average RV from all the spectral lines on a wavelength interval $\lambda 4400-5100 \AA$.

It is well known that individual spectral lines of roAp stars show RV amplitudes that differ by up to factors of 100 or more. Indeed, the analysis of individual spectral lines have yielded important information about the oscillations in roAp stars including the presence of radial nodes in the stellar atmosphere (e.g. Mkrtichian et al. 2003; Kurtz et al. 2005;
Ryabchikova et al. 2007b). However, investigations of the mean $\mathrm{RV}$ variations of all spectral lines can also produce important results that can be missed by studies concentrating only on a few spectral lines. Such averaging of RV measurements in the case of minor phase differences between individual lines can suppress the noise and significantly increase the precision of the RV measurements, making the detection of low amplitude modes possible. It was these "integral" RV measurements that revealed the roAp nature of $\beta \mathrm{CrB}$ (Hatzes \& Mkrtichian 2004).

The detailed line-by-line examination of the RV variations of individual chemical elements and their ions will be the subject of a forthcoming paper of this series on HD 101065.

\section{Observations and data reduction}

Spectroscopic observations of HD 101065 were obtained on the nights of 3-6 March 2004 at the European Southern Observatory (ESO) under program 072.D-0286 using the HARPS echellespectrometer of the 3.6-m telescope. The instrument provides a resolving power $R(=\lambda / \Delta \lambda)=110000$ with a wavelength coverage of 4700-7400 $\AA$. Exposure times were typically $60 \mathrm{~s}$ with a readout time of about $30 \mathrm{~s}$. Typical signal-to-noise ratios $(S / N)$ were 20-30 per pixel, depending on transparency, seeing, etc. Observations were performed using an iodine absorption cell to provide the wavelength reference in the spectral range 5200-6000 A. We opted for use of the cell because we anticipated doing a line-by-line RV analysis. At the time HARPS was recently commissioned and it was not clear how well the ThAr calibration could be used for such an analysis. Unlike ThAr emission lines, molecular iodine has a rich forest of lines that could be used for monitoring the instrumental shifts and other variations (instrumental profile, for example) over a narrow wavelength range. (Two weeks after our run the iodine cell broke and is no longer available on HARPS.) Since then it has been established that the nightly instrumental drifts of HARPS are less than $1 \mathrm{~m} \mathrm{~s}^{-1}$, more than adequate for studying the short period oscillations of HD 101065. Because we used the iodine cell we were not able to use the simultaneous Th-Ar calibration. All wavelength calibrations outside of the iodine regions were performed using the Th-Ar calibration taken at the beginning and end of the night, but since the nightly drifts are less than the amplitude of our detected modes only a few calibration frames are sufficient.

The Image Reduction and Analysis Facility (IRAF) was used to perform the standard reduction procedures of bias subtraction, global scattered subtraction, and flat-fielding to remove pixel-to-pixel variations. Spectral orders were extracted using the "echelle" package of IRAF.

Precise stellar radial velocity measurements were obtained for all spectral lines in the two wavelength regions: 14400-5100 $\AA$ that were free from iodine lines (hereafter the "blue" range) and the wavelength interval $25200-5800 \AA$ that covered regions where iodine absorption lines were reasonably strong (hereafter the "green" range). In the wavelength range 15800-6200 A there are iodine lines present, but these are somewhat weak for use as wavelength reference compared to other regions and were thus not used. The wavelength range of $\lambda 6300-$ $7400 \AA$ was also relatively clear of iodine absorption lines, but this region is often contaminated with telluric lines so it is not suitable for "integrated" RV measurements.

For the "out-of-iodine" wavelength regions we used a modified version of the iodine cell reduction package. When reducing "iodine cell" data the routine divides each spectral order into 
Table 1. Log of the February 27-March 5, 2004 spectroscopic (ESO) and photometric (Siding Spring Observatory) two-site campaigns. The fourth column shows the number of $40 \mathrm{~s}$ integrations for photometry and the number of RV data points per night.

\begin{tabular}{llllll}
\hline \hline Date & \multicolumn{2}{c}{ HJD 2 453000+ } & $N$ & Obs./Tel. & Filter/Spectrograph \\
& Start & End & & & \\
\hline 26 Feb. 04 & 62.0874 & 62.1415 & 60 & SSO/24" & $U$ \\
27 Feb. 04 & 63.0636 & 63.2221 & 291 & SSO/24" & $U$ \\
29 Feb. 04 & 64.9933 & 65.1026 & 203 & SSO/24" & $U$ \\
02 Mar. 04 & 66.9047 & 67.0036 & 165 & SSO/24" & $B$ \\
03 Mar. 04 & 67.6053 & 67.8875 & 238 & ESO/3.6m & HARPS \\
03 Mar. 04 & 67.9133 & 67.9749 & 107 & SSO/24" & $B$ \\
04 Mar. 04 & 68.5610 & 68.8888 & 296 & ESO/3.6m & HARPS \\
04 Mar. 04 & 68.9194 & 69.0236 & 157 & SSO/24" & $B$ \\
05 Mar. 04 & 69.5816 & 69.8934 & 264 & ESO/3.6m & HARPS \\
06 Mar. 04 & 70.5776 & 70.8960 & 276 & ESO/3.6m & HARPS \\
\hline
\end{tabular}

individual chunks, in this case 10 chunks per order. In each spectral chunk an observation is matched to a template reference spectrum taken at high $\mathrm{S} / \mathrm{N}$ ratio. This is done by varying a large number of parameters which include a second order fit to the local continuum (thus a local normalization is employed), the coefficients of the wavelength solution, the functions defining the instrumental profile (typically 5 Gaussian profiles), and the relative shift between the template reference spectrum (in the chunk) and the data spectrum. The latter was done using the shift-detection algorithm of Fahlmann \& Glaspey (1973). The main difference for the HARPS Th-Ar analysis is that there is no superimposed iodine reference spectrum for which to determine both the wavelength scale and the instrumental profile (see Valenti et al. 1995, for a desciption of how this is done). Instead the wavelength solution as determined by a Th-Ar calibration lamp is kept fixed and the instrumental profile is not varied during the RV calculation. This is perfectly valid for HARPS data as the extreme stability of the spectrograph ensures that the instrumental profile remains constant and that there are no changes in the wavelength scale throughout the night or from night-to-night. Afterwards, the relative RVs from the individual chunks were averaged to produce a single RV value for each observation. The typical accuracy of individual measurements was $3-4 \mathrm{~m} \mathrm{~s}^{-1}$ as measured by the rms scatter of the RVs from the individual chunks.

Comparison of the detection threshold between "blue" and "green" radial velocity measurements revealed a significantly better detection threshold for the "blue" RVs with respect to the those from the iodine regions primarily because of the increased line density in the blue regions of the spectra. The blue spectral regions had roughly $2-3$ times the line density over the green spectral regions and this resulted in an improvement in the RV accuracy by a factor of $\approx 1.5$ or more. Although the iodine cell causes some reduction of light, this is made up by the fact that the spectrograph efficiency is higher towards the red. Thus the signal-to-noise ratios in the blue and green regions were comparable. In the analysis presented below we used only the RV measurements from the "blue" spectral regions.

The supporting Johnson $U$ and $B$ photometric observations of HD 101065 were acquired on three nights between 27-29 April 2004 and on three consecutive nights 2-4 March 2004 using the Motorized Filter Box photometer attached to a 24-inch telescope at Siding Spring Observatory (Australia). Photometric apertures of 30-40 arcsec were used, depending on the telescope's tracking performance and seeing. The standard rapid photometry reduction procedures were applied using the comparison star HD $101066(V=7.81, B-V=0.31)$. The longterm atmospheric transparency variations were removed using high-order polynomials in time. Finally, the 10-s integrations were binned to 40-s integrations to reduce the noise. The photometric light curves covered the time interval from before the spectroscopic run to just before the last two nights of the spectroscopic run. This provided accurate photometric phase determination for the principal mode.

The combined journal of photometric and spectroscopic observations is given in Table 1 which lists the JD dates of the observing sequences, the number of spectra, and photometric data points per night.

\section{The analysis}

A previous photometric investigation of HD 101065 (Martinez \& Kurtz 1990) showed a modulation for the principal photometric mode with a period of a few days. In roAp stars the strictly periodic modulation of amplitudes and phases of individual modes was explained within the framework of the "oblique pulsator model" and is the result of the changing rotational aspect of the axis of symmetry of nonradial modes which is aligned with the magnetic axis (Kurtz 1990). The preliminary analysis of RV data obtained on the $15200-6000 \AA$ A sub-interval (Mkrtichian \& Hatzes 2005) was governed by the assumption that the nightly variations were due to the rotational modulation of the star. Based on that assumption the authors fitted a sine function to the principal mode in order to find the amplitude and phase for the principal oscillation mode. After removing this best sine-wave fit to the data of individual nights the merged residuals were used to search for additional low-amplitude modes. This preliminary analysis revealed five excited modes. Meanwhile, we will show below that the observed amplitude modulation is actually a beating of modes closely spaced in frequency and is not due to rotational modulation. These closely spaced modes were actually lost in the preliminary analysis.

The oscillation spectrum of HD 101065 derived in this section is based on other RV measurements using all spectral features in the 4400-5100 ̊ wavelength interval. Since many spectral lines can pulsate $180^{\circ}$ out-of-phase with most other spectral features (Baldry et al. 1998; Mkrtichian et al. 2003) there is some concern that using integrated RV measurements of many lines would reduce the observed pulsational amplitude making it more difficult to detect low amplitude modes. This seems not to be the case for HD 101065. Figure 1 shows the measurements of principal mode pulsation semi-amplitude and phase for individual spectral lines plotted with respect to the equivalent width. Each data point represents binned averages (phase bins of $10 \mathrm{~m} \AA$ were used) of over 400 spectral lines. As seen, the oscillation phase of the majority of the spectral lines is only slightly variable within \pm 0.02 of pulsation period, 


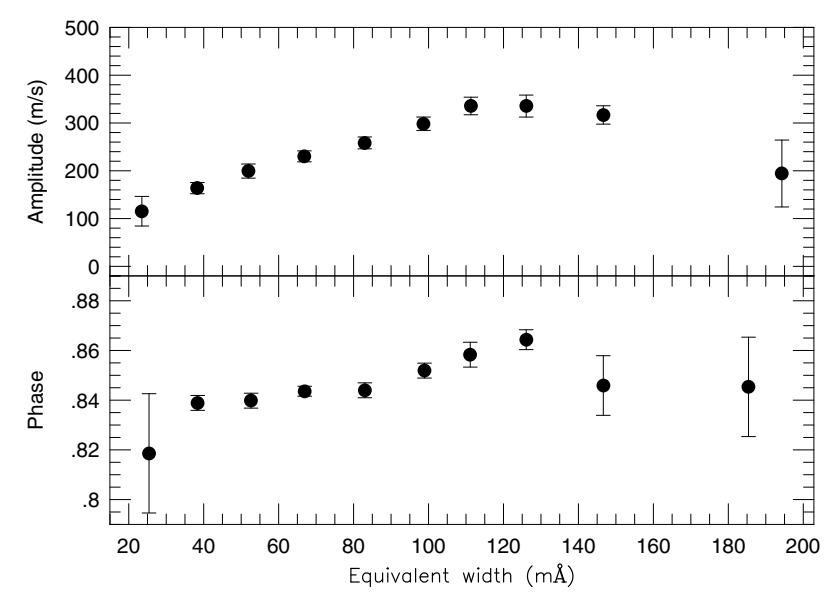

Fig. 1. The phase-binned averages of the pulsational semi-amplitude (top panel) and phase (bottom panel) of individual lines as a function of the equivalent width for HD 101065. Over 400 spectral lines of various atomic species were used in the binned averages

thus "integral" radial velocities should be able to achieve the best possible RV precision. This increased precision results in the detection of many low-amplitude modes. Note that pulsation amplitude and phase for individual spectral lines in HD 101065 were recently studied by Ryabchikova et al. (2007b) based on re-analysis of $2 \mathrm{~h}$ duration spectroscopic time series obtained at VLT in the context of the observing program 072.D-0138 (Kurtz et al. 2006). They found that the pulsation phase does not change by more than 0.15 for the majority of spectral lines, a result that is consistent with ours. They found that rapid change in pulsation phase occurs in Tb III followed by Th III lines that are formed in the upper atmospheric layers.

The "blue integral" radial velocity (RV) curves are shown in Fig. 2 by dots. An example of the photometric light curves obtained in the $U$ filter on the two consecutive nights 26 and 27 February 2004 are shown in Fig. 3 The time series analysis of RV and photometric data was performed using discrete Fourier transform (DFT) and a non-linear least-square fitting program Period04 (Lenz \& Breger 2005). The "classical" consecutive pre-whitening procedure was employed. This sequentially removes the contribution of the highest amplitude mode after subtracting the contribution of the previous dominant frequency.

The steps of consecutive DFT analysis of the merged JD 2453 067-JD 2453070 blue RV data set are shown in the panels of Figs. 4 and 5 from top to bottom. Note that the amplitude scale in the figures changes drastically from top to bottom. Through this sequential pre-whitening procedure we found 26 periodic signals in the data. Among these 15 belong to a modal spectrum, one is probably an artifact, while the remaining signals are the first and second harmonics of oscillation modes. The power spectrum of a signal in the data of single-site observations is affected by the window function which leads to peaks in the power spectrum at a $\pm 11.574 \mu \mathrm{Hz}$ alias of the frequency of the real signal. An analysis of possible frequency mismatches in the DFT spectrum was done by comparing the frequencies from the analysis using the spacing of $\Delta v_{0} / 2=32 \mu \mathrm{Hz}$ found for most of the modes. We identified two peaks that differed by $11.574 \mu \mathrm{Hz}$ from frequencies consistent with the spacing of $\Delta v_{0}$ and two that were aliases of high-frequency harmonics of a real signal. The frequencies found in the HD 101065 data and their uncertainties calculated using the Monte-Carlo simulation option of the Period04 program (Lenz \& Breger 2005) are given in Table 2. The oscillation frequencies optimized for aliases are marked by bullets. The detection threshold in the DFT spectrum, in the bottom panel of Fig. 4 in the frequency range of interest of $1300-2000 \mu \mathrm{Hz}$ is about $1.1 \mathrm{~m} \mathrm{~s}^{-1}$. The excess signal (1.1$1.7 \mathrm{~m} \mathrm{~s}^{-1}$ ) between $2700-2800 \mu \mathrm{Hz}$ and $4000-4200 \mu \mathrm{Hz}$ may be due to the unresolved spectrum of the first and the second harmonics of other low-amplitude modes.

Among the frequencies from Table 2, five were already found in a preliminary RV analysis of our spectroscopy using a wavelength sub-interval of $15000-6200 \AA$ (Mkrtichian \& Hatzes 2005). Two, $f_{1}=1372.8 \mu \mathrm{Hz}$ and $f_{3}=1314.6 \mu \mathrm{Hz}$, are near the frequencies $\left(v_{1}=1372.89 \pm 0.94 \mu \mathrm{Hz}\right.$ and $v_{3}=1315.07 \pm$ $0.94 \mu \mathrm{Hz}$ ) found earlier in the photometric analysis by Martinez $\&$ Kurtz (1990). One more frequency at $\nu_{2}=1369.63 \mu \mathrm{Hz}$ that was reported by Martinez \& Kurtz (1990) can now be interpreted as a $1 \mathrm{c} \mathrm{d}^{-1}(11.574 \mu \mathrm{Hz})$ alias of the $f_{2}=1381.47 \mu \mathrm{Hz}$ frequency found in our analysis.

The analysis of the photometric data of the $U$-band data $(\mathrm{JD}=2453062-2453064)$ and the three consecutive nights of $B$-band observations made before and during the spectroscopic observations revealed only the principal mode oscillation at $f_{3}=1372 \mu \mathrm{Hz}$. The mean photometric phase and amplitude of the principal mode was determined by sine wave fit to the data. The resulting $U$ and $B$ mean semi-amplitudes, moments of light maxima, phase shifts compared to RV-maximum, and velocity-to-magnitude ratio are given in Table 3. In summary, our spectroscopic analysis using only four nights of ground-based HARPS spectroscopy revealed modes with semiamplitudes ranging from $218 \mathrm{~m} \mathrm{~s}^{-1}$ to $1.6 \mathrm{~m} \mathrm{~s}^{-1}$, or more than a factor of 100 difference in amplitude. This beautifully demonstrates the advantages of spectroscopy over photometry in the study of oscillations in roAp stars.

\section{P-mode spacing and echelle-diagram}

The schematic of the RV oscillation spectrum for HD 101065 is shown in Fig. 6. One can clearly see a regular large spacing with a separation of about $32 \mu \mathrm{Hz}$ and a small spacing of about 3-7 $\mu \mathrm{Hz}$. The regular frequency spacing in the oscillation spectra of solar-type and roAp stars is a signature of the excitation of consecutive overtones of high overtone p-modes. The "large frequency spacing" is the spacing between the consecutive overtones of modes of the same degree: $\Delta v=f(n, \ell)-f(n-1, \ell)$, where $n$ is radial order and $\ell$ the degree of the mode. The "small spacing" is the frequency difference of modes of the same parity $\delta v_{\ell}=f(n, \ell)-f(n-1, \ell+2)$. The presence of both the large and small frequency spacings in the HD 101065 oscillation spectrum is an indicator of excitation of consecutive overtones over the range of low $\ell$ p-modes. The observed value of the mean "large separation" $\Delta v=64.07 \pm 0.91 \mu \mathrm{Hz}$ was determined as a mean frequency spacing of all frequencies expected to be consecutive overtones of modes of equal degree. The echelle-diagram plotted with $\Delta v=64.0 \mu \mathrm{Hz}$ spacing is shown in Fig. 9 (dots). This more clearly shows the regular large and small spacing of frequencies grouped in vertical columns.

In the roAp stars the magnetic field of a few $\mathrm{kG}$ can change the pulsation frequency by $\approx 10-30 \mu \mathrm{Hz}$, depending on latitudinal degree $\ell$. The frequencies of $\ell=0$ and $\ell=3$ modes tend to be affected more strongly (Saio 2005). Thus the resulting frequency spacings can be quite different from those expected without a magnetic field. The global magnetic field can thus be determined by comparing the observed oscillation frequencies to predicted values of the model with magnetic fields. 


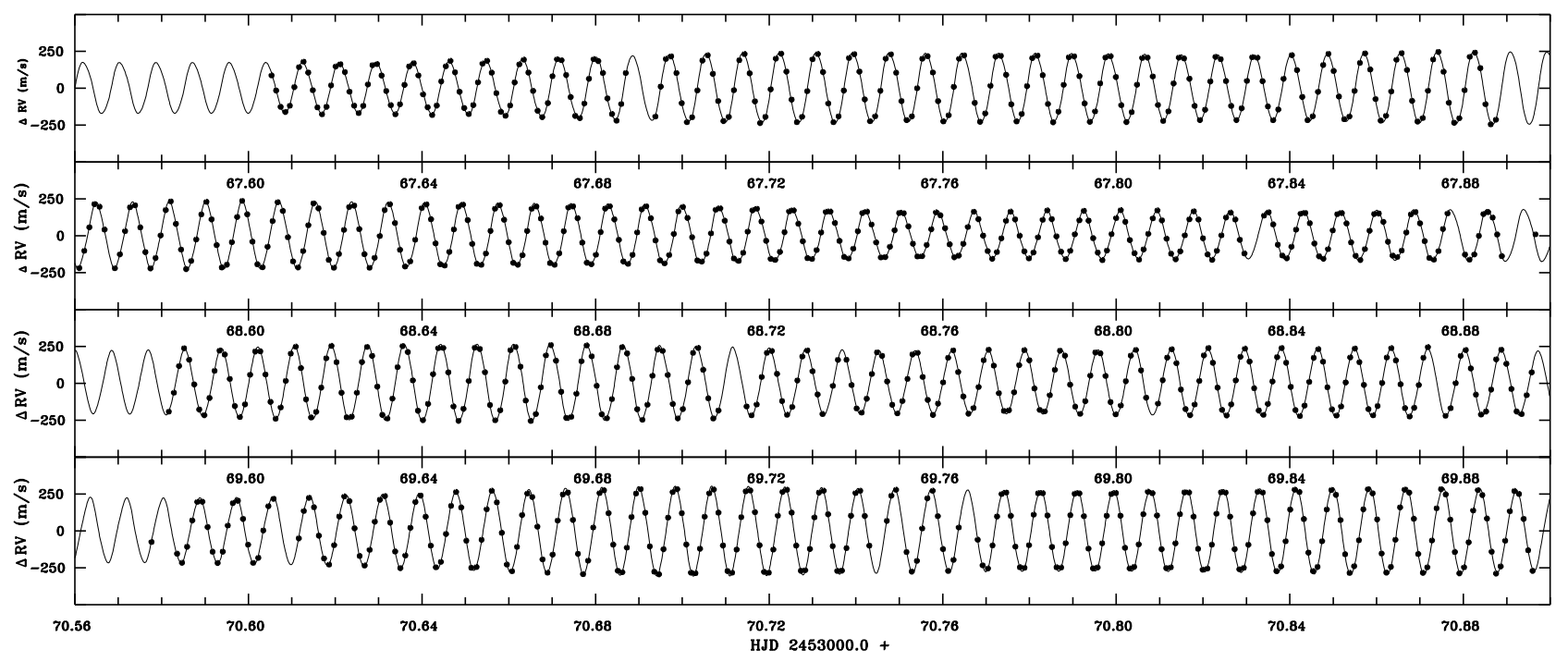

Fig. 2. The pulsational radial velocity curves of HD 101065. The solid line is the synthetic multifrequency fit.

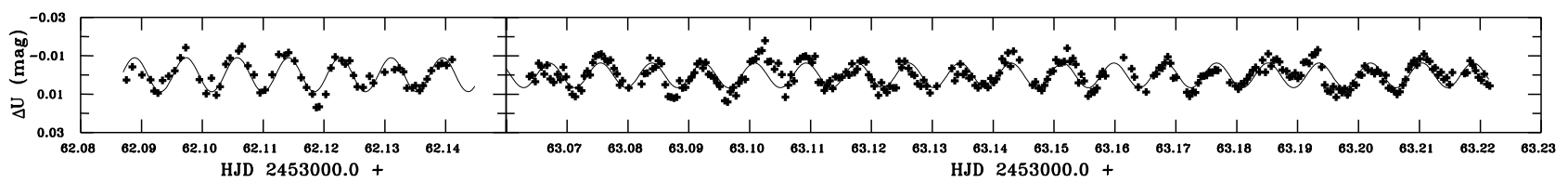

Fig. 3. The pulsational U filter photometric curve on the two consecutive nights of 26 and 27 February 2004 for HD 101065. The solid lines are the nightly synthetic fit for $f_{1}$ mode.

\section{Modelling of p-mode spectrum and asteroseismic calibrations of HD 101065}

We have obtained main-sequence evolution models for stellar masses ranging from $1.5 M_{\odot}$ to $1.7 M_{\odot}$ with an increment of $0.05 M_{\odot}$. Two set of chemical compositions, $(X, Z)=(0.7,0.02)$ and $(0.695,0.025)$ were adopted, where $X$ and $Z$ are the mass fractions of hydrogen and heavy elements, respectively. The opacity was obtained from OPAL opacity tables (Iglesias \& Rogers 1996). It was assumed that a strong magnetic field suppresses convection completely in the envelope and that helium is depleted above the He I ionization zone as $Y=0.01+0.27\left(x_{2}+\right.$ $x_{3}$ ) (cf. Balmforth et al. 2001), where $Y$ is the mass fraction of helium, and $x_{2}$ and $x_{3}$ are fractions of singly and doubly ionized helium, respectively. Above the photosphere, the $T-\tau$ relation given in Shibahashi \& Saio (1985) was used. Figure 7 shows the evolutionary tracks of our models and the location of HD 101065 (open circle) with error bars adopted from the estimation by Kochukhov \& Bagnulo (2006).

We have calculated nonadiabatic frequencies of axisymmetric high order $\mathrm{p}$-modes under dipole magnetic fields. Under the influence of a magnetic field, the angular dependence of a pulsation mode deviates from that of a single spherical harmonic. We have employed the calculation method discussed in Saio (2005), in which the angular dependence of pulsations is expressed by a sum of terms proportional to spherical harmonics of various $\ell ; Y_{\ell}^{m=0}$. Pulsation modes are classified into two mutually independent groups: even and odd modes. An even mode is expressed by a sum of components associated with $\ell=0,2,4, \ldots$; while an odd mode with $\ell=1,3,5, \ldots$ We have usually included 12 (occasionally 14) components for each mode. The contribution from each of the components associated with different $\ell$ differs from mode to mode, and changes when the magnetic field strength varies. We designate the character of latitudinal dependence of a mode by $\ell_{\mathrm{m}}$ that indicates the $\ell$ value of the component whose kinetic energy is largest among the components of expansion. (The value of $\ell_{\mathrm{m}}$ can shift by 2 as the strength of magnetic field changes.)

The outer boundary condition is imposed at an optical depth of 0.001 . We have used a reflective outer boundary condition; all the obtained frequencies are smaller than the critical frequencies.

Figure 8 shows the high-order $(18 \leq n \leq 23)$ p-mode frequencies of various $\ell_{\mathrm{m}}$ as a function of $B_{\mathrm{p}}$ (the polar strength of the magnetic field) for a model of $1.55 M_{\odot}$ with $(X, Z)=$ $(0.7,0.02)$. The observed frequencies of HD 101065 are shown by horizontal dashed lines. The theoretical frequencies of this model agree reasonably well with the observed frequencies at $B_{\mathrm{p}} \approx 9 \mathrm{kG}$. Unfortunately, we have found no excited modes in our analysis for the appropriate frequency range. The discrepancy in the mode stability may come from our simple diffusion approximation used for the radiation flow even above the photosphere, although the atmospheric part seems to considerably affect the stability of high-order p-modes. In the following we discuss the pulsation frequencies.

Generally, the pulsation frequency of a mode increases as $B_{\mathrm{p}}$ increases without changing much the frequency interval between two adjacent order modes (the large separation of frequencies). At some values of $B_{\mathrm{p}}$, however, the kinetic energy associated with components $\ell$ greater than $\ell_{\mathrm{m}}$ becomes larger so that the expansion for the eigenfunction fails to converge or $\ell_{\mathrm{m}}$ shifts to a larger value. At a similar value of $B_{\mathrm{p}}$ a new sequence starts and extends to higher $B_{\mathrm{p}}$ at a slightly smaller frequency. Such transitions occur cyclically in terms of $B_{\mathrm{p}}$ for a given mode or in terms of frequencies at a given $B_{\mathrm{p}}$ (Cunha \& Gough 2000; Saio \& Gautschy 2004). In the case shown in Fig. 8, these transitions occur around $2 \mathrm{kG}$ and $7 \pm 1 \mathrm{kG}$.

Interestingly, this figure shows that in such a transition occurring around $B_{\mathrm{p}}=7 \pm 1 \mathrm{kG}$, two modes associated with 


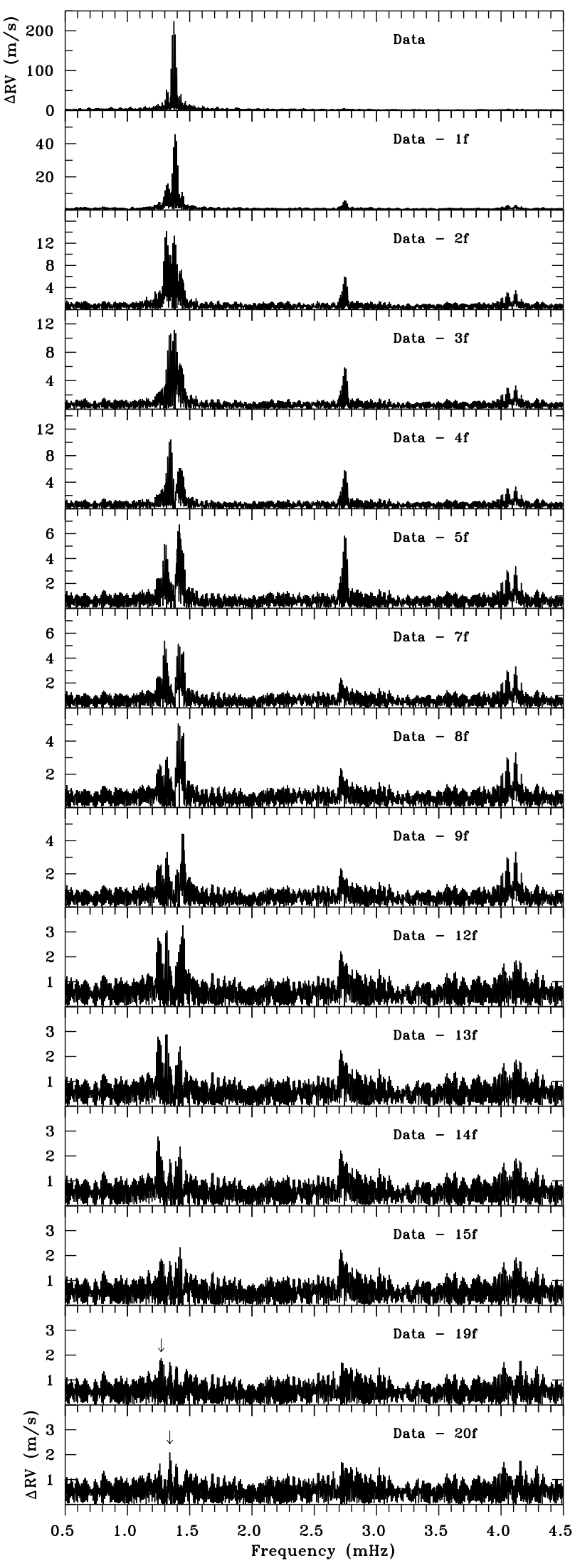

Fig. 4. The selected amplitude spectra of consecutive steps of a DFT analysis for the original RV data. In bottom panels the position of the largest peaks are shown by arrows. Note that the amplitude scale decreases by about a factor of 60 from the top to bottom panels. Due to space restrictions we do not plot the DFT spectra showing the removal of the first and the second harmonics of oscillation frequencies.

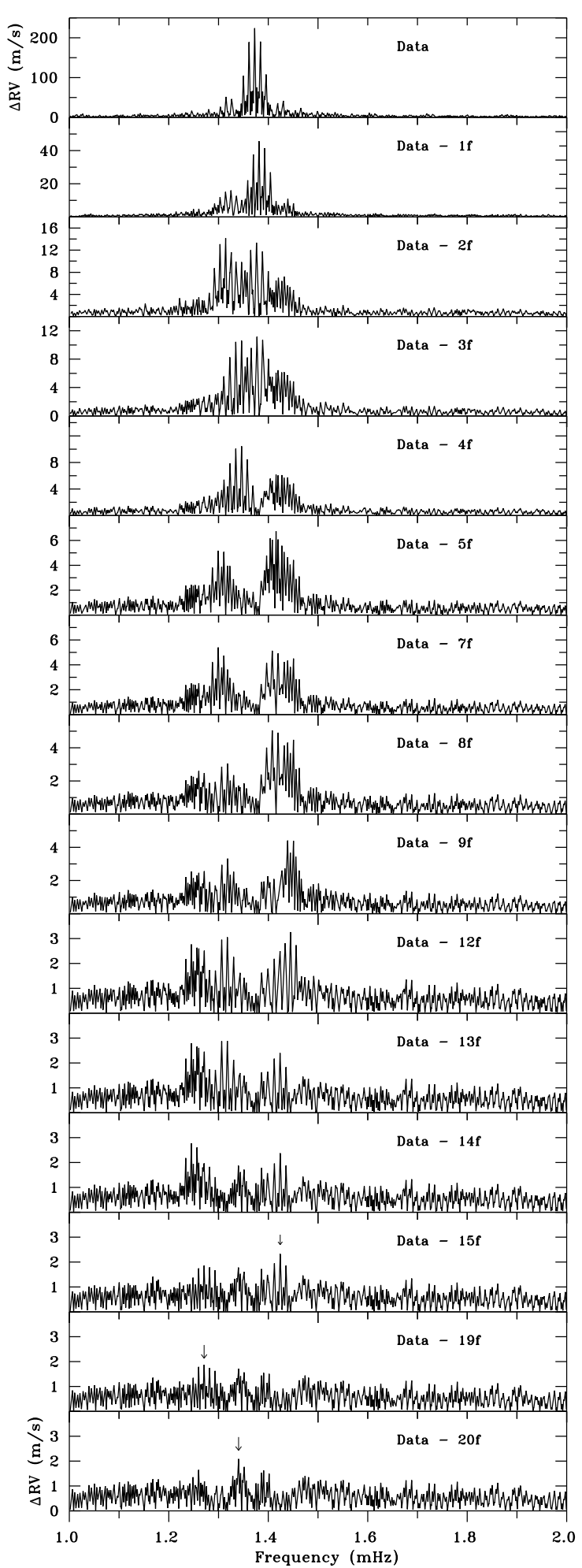

Fig. 5. The same as in Fig. 4 but shown in frequency range $1-2 \mu \mathrm{Hz}$.

the same radial order and $\ell_{\mathrm{m}}$ appear in a certain range of $B_{\mathrm{p}}$ for $\ell_{\mathrm{m}}=1$ and 2 . The two modes having the same radial order and $\ell_{\mathrm{m}}$ have different frequencies because the distribution of kinetic energy between the $\ell$-components of the eigenfunctions are different. These additional frequencies appear roughly in the middle of the large separation for each $\ell_{\mathrm{m}}$ and form closely separated frequency groups with modes of different $\ell_{\mathrm{m}}$. Such groups 
Table 2. The frequencies and semi-amplitudes of signals detected in the integral $\lambda 4400-5100 \AA \mathrm{RV}$ data. The nomenclature of frequencies is given according to the order of their detection in the DFT spectrum. The bullets denote two frequencies optimized for $11.574 \mu \mathrm{Hz}$ mismatches, the circle denotes a frequency that probably is not an oscillation mode.

\begin{tabular}{llll}
\hline \hline No. & Frequency & $K$ & Phase \\
& $\mu \mathrm{Hz}$ & $\mathrm{m} \mathrm{s}^{-1}$ & \\
\hline$f_{1}$ & $1372.80 \pm .004$ & $217.9 \pm .49$ & $0.7682 \pm .0004$ \\
$f_{2}$ & $1381.47 \pm .02$ & $43.8 \pm .49$ & $0.662 \pm .002$ \\
$f_{3}$ & $1314.57 \pm .06$ & $14.9 \pm .50$ & $0.219 \pm .005$ \\
$f_{4}$ & $1379.80 \pm .06$ & $13.3 \pm .49$ & $0.109 \pm .006$ \\
$f_{5}$ & $1346.22 \pm .07$ & $12.0 \pm .47$ & $0.278 \pm .006$ \\
$f_{6}$ & $1415.76 \pm .13$ & $6.6 \pm .48$ & $0.062 \pm .012$ \\
$f_{7}$ & $2743.98 \pm .14$ & $5.9 \pm .47$ & $0.886 \pm .012$ \\
$f_{8}$ & $1287.38 \pm .16 \bullet$ & $5.2 \pm .47$ & $0.027 \pm .015$ \\
$f_{9}$ & $1407.92 \pm .14$ & $5.9 \pm .46$ & $0.152 \pm .013$ \\
$f_{10}$ & $1439.01 \pm .14 \bullet$ & $6.1 \pm .55$ & $0.530 \pm .014$ \\
$f_{11}$ & $1318.06 \pm .22$ & $3.9 \pm .47$ & $0.079 \pm .019$ \\
$f_{12}$ & $4116.92 \pm .20$ & $4.3 \pm .49$ & $0.734 \pm .018$ \\
$f_{13}$ & $4059.05 \pm .22$ & $3.9 \pm .47$ & $0.323 \pm .019$ \\
$f_{14}$ & $1444.96 \pm .27$ & $3.2 \pm .47$ & $0.136 \pm .024$ \\
$f_{15}$ & $1245.46 \pm .32$ & $2.6 \pm .46$ & $0.575 \pm .028$ \\
$f_{16}$ & $2711.76 \pm .36$ & $2.3 \pm .44$ & $0.352 \pm .032$ \\
$f_{17}$ & $1473.99 \pm .28$ & $3.1 \pm .56$ & $0.137 \pm .029$ \\
$f_{18}$ & $1351.66 \pm .34$ & $2.5 \pm .49$ & $0.947 \pm .031$ \\
$f_{19}$ & $2758.37 \pm .39$ & $2.2 \pm .44$ & $0.581 \pm .036$ \\
$f_{20}$ & $1424.22 \pm .39 \circ$ & $2.2 \pm .47$ & $0.389 \pm .035$ \\
$f_{21}$ & $4120.90 \pm .42$ & $2.0 \pm .47$ & $0.058 \pm .036$ \\
$f_{22}$ & $1282.15 \pm .43$ & $2.0 \pm .46$ & $0.767 \pm .040$ \\
$f_{23}$ & $4150.87 \pm .45$ & $1.9 \pm .45$ & $0.520 \pm .040$ \\
$f_{24}$ & $4024.01 \pm .47$ & $1.8 \pm .46$ & $0.918 \pm .043$ \\
$f_{25}$ & $2719.12 \pm .46$ & $1.9 \pm .47$ & $0.134 \pm .041$ \\
$f_{26}$ & $4082.01 \pm .47$ & $1.6 \pm .45$ & $0.556 \pm .041$ \\
\hline & & &
\end{tabular}

having small separations around the middle of a large separation are needed to explain the observed frequencies of HD 101065. For the model shown in Fig. 8, theoretical frequencies are within $\pm 1.3 \mu \mathrm{Hz}$ consistent with the observed ones around $B_{\mathrm{p}} \approx 9 \mathrm{kG}$.

For two or three stellar models for each mass, we have obtained theoretical frequencies in the observed frequency range for $2 \leq B_{\mathrm{p}} / \mathrm{kG}<10$ with an interval of $0.2 \mathrm{kG}$ as shown in Fig. 8. The effective temperature (and luminosity) that gives frequencies most consistent with the observed ones was obtained by interpolating between two models on the evolutionary track of a given mass. The goodness of fit of a model is judged by the mean deviation between the observed and model frequencies, in which we have used the logarithmic values of amplitudes $\left(\mathrm{m} \mathrm{s}^{-1}\right)$ as weight. The results of this exercise are given in Table 4 . The smallest mean deviation was obtained for $1.55 M_{\odot}$ with $(X, Z)=(0.7,0.02)$, although the difference is small among the cases considered. The loci of the best-fit model for each mass on the HR diagrams are shown in Fig. 7 connected by dotted lines for both chemical compositions. Obviously, the loci are insensitive to the heavy-element abundance, because the location is mainly determined by the large separation of frequencies that is a function of mass and radius.

Table 5 shows the detailed comparison between the observed and theoretical frequencies for the two best fitting models of $1.55 M_{\odot}$ and $1.50 M_{\odot}$ with $Z=0.02$, in which $n$ indicates the radial order of a mode. Figure 9 shows echelle diagrams of the best fit models with the observed frequencies of HD 101065. The required magnetic field strength to fit the observed frequencies tends to be smaller for the more massive models. Since the mean density of the envelope is larger for less massive models, a stronger magnetic field is required to have similar effects on the pulsation frequencies of the less massive models. It is remarkable that most of the theoretical frequencies for $\ell_{\mathrm{m}}<3$ correspond to observed frequencies.

In summary, based on the detected rich multi-mode oscillation spectrum and theoretical modelling we find the following asteroseismically determined stellar parameters for HD 101065: stellar mass, $M=1.525 \pm 0.025 M_{\odot}$, Age $=(1.5 \pm 0.1) \times 10^{9} \mathrm{yr}$, effective temperature, $\log T_{\mathrm{e}}=3.821 \pm 0.006$, luminosity, $\log L / L_{\odot}=0.797 \pm 0.026, \log g=4.06 \pm 0.04$, and magnetic field strength, $\mathrm{Bp}=8.7 \pm 0.3 \mathrm{kG}$. The accurate location of HD 101065 is shown in Fig. 7 by open squares with error bars. The accuracy of our stellar parameters was limited by the accuracy of frequency determinations which was governed by the 4-day observation interval. Further progress in modelling requires an accuracy in the pulsation frequency determinations to much better than $1 \mu \mathrm{Hz}$ and by improving the stellar models.

\section{Discussion}

\subsection{The rotation period and long-term pulsation amplitude variability}

Magnetic fields measurements for HD 101065 over the last 4 decades have been rare (Wolff \& Hagen 1976; Cowley \& Mathys 1998; Hubrig et al. 2004). Among them, the photographic measurements by Wolff \& Hagen (1976) were obtained with a lower dispersion $\left(13.4 \AA \mathrm{mm}^{-1}\right.$ ) than is usual for Zeeman observations and should be revised now. These three magnetic field measurements of $-2100,-2100$, and $-2500 \mathrm{G}$ had a standard deviation of $\pm 450 \mathrm{G}$. Fortunately, they obtained two similar measurements of non-magnetic Am star $\delta$ Nor $B_{1}=-290 \mathrm{G}$ and $-320 \mathrm{G}$ with a standard deviation $\pm 270 \mathrm{G}$, while measurements with a dispersion $6.7 \AA \mathrm{mm}^{-1}$ show a mean field $+16.6 \pm 80 \mathrm{G}$ that is close to zero within errors. The mean value of $-305 \mathrm{G}$ measured for a non-magnetic star is most likely an instrumental shift for a low resolution measurements of HD 101065 and should be subtracted. Another point of concern is that lacking good atomic data, Wolff \& Hagen used an average effective Lande factor 1.0 for the magnetic measurement in the 4000-4700 $\AA$ wavelength range which contained about 45 spectral lines and blends of La II, Ce II, Nd II, Sm II, Dy II and Ho II (the wavelengths of these lines were not listed). However, investigations of the distribution of Lande factors for Ap star lines in photograpic wavelength regions show an average value of $g_{\mathrm{eff}}=1.23$. About $85 \%$ of lines have Lande factors ranging from $g_{\mathrm{eff}}=0.8$ to $g_{\mathrm{eff}}=1.5$, and about half of the lines with $g_{\text {eff }}$ between 1.0 and 1.5 (Romanuyk 1984). Thus, magnetic field values by Wolff \& Hagen, corrected for the instrumental shift, should also be reduced by about $23 \%$. This results in a revised mean value of three magnetic field measurements of $B_{1}=1567 \pm 210 \mathrm{G}$.

Mean magnetic field measurements are compiled in Table 6 and are shown in Fig. 10 (top panel). These data show clear evidence of a long-term decrease in the magnetic field strength at a rate of $-18 \mathrm{G} \mathrm{yr}^{-1}$; however, there are insufficient measurements to draw any conclusions about possible periodic variability. In Fig. 10 (middle panel) we plot the mean $B$-filter pulsation semi-amplitude over the nights of 2-4 March 2004 along with the $B$ semi-amplitude for other epochs measured by Martinez $\&$ Kurtz (1990). The bottom panel shows the expected angle $\alpha$ between magnetic pole and line of sight calculated for polar 
Table 3. The semi-amplitudes, epochs of the $U, B$ light and RV maxima of the principal $f_{1}$ mode. The last column gives phase shifts between RVs maximum and light curve minimum.

\begin{tabular}{llccc}
\hline \hline Quantity & Semi-amplitude & $\begin{array}{c}\text { Epoch of max. } \\
2453000 .+\end{array}$ & $\begin{array}{c}2 K / \Delta m \\
\mathrm{~km} \mathrm{~s}^{-1} \mathrm{mag}^{-1}\end{array}$ & Phase lag \\
\hline$\Delta U$ & $6.22 \pm 0.27 \mathrm{mmag}$ & $67.79665 \pm 0.00006$ & 35.1 & $0.156 \pm 0.007$ \\
$\Delta B$ & $4.85 \pm 0.33 \mathrm{mmag}$ & $67.79635 \pm 0.00009$ & 45.0 & $0.192 \pm 0.011$ \\
$\Delta R V$ & $218.4 \mathrm{~m} \mathrm{~s}^{-1}$ & $67.79797 \pm 0.000003$ & - & - \\
\hline
\end{tabular}

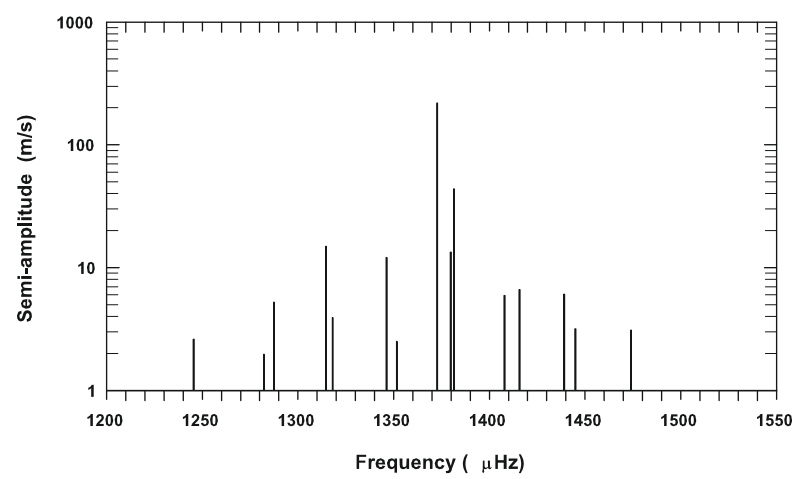

Fig. 6. The schematic of the oscillation spectrum of HD 101065. The two small and large frequency spacings in the oscillation spectrum are well visible.

magnetic field strength $B_{\mathrm{p}}=8.7 \mathrm{kG}$ using the well known formula for a dipole magnetic field (Babcock 1958)

$\cos \alpha=\frac{B_{1}}{B_{\mathrm{p}}} \frac{20(3-u)}{15+u}$

with $u$ being the limb darkening coefficient (taken as 0.616 ).

As can be seen in Fig. 10, a change in the inclination of the magnetic axis from about $56^{\circ}$ to $69^{\circ}$ (corresponding variations of $\cos \alpha$ from 0.56 to 0.36 ) led to a decrease of apparent magnetic field by about $35 \%$. Over a quarter of a century (note the 16 year gap between our observations and older ones), the $B$ semi-amplitude ranged within $4.94-5.76$ mag (about a $15 \%$ variation) and likely varied irregularly from season to season, as was earlier found for shorter intervals of observations by Martinez \& Kurtz (1990). Again, there is not enough information about photometric mode variability to give any strong conclusion about the correlation between magnetic field and pulsational amplitude changes in HD 101065.

Below, we calculated the expected changes of amplitudes of pulsation modes with the stellar rotation and aspect changes of the magnetic pole. These should be very useful for confirmation of mode identification in the future when more seasonal measurements of magnetic field and modal pulsation amplitudes of HD 101065 will be collected. The interaction with the magnetic field makes the latitudinal dependence of the pulsational amplitude deviate from that of $P_{\ell_{\mathrm{m}}}(\cos \theta)$, partitioning pulsation energy into components other than the main $\left(\ell=\ell_{\mathrm{m}}\right)$ one (Saio $\&$ Gautschy 2004). The phase relation in the distribution of displacements and flux variation on the surface might be quite complicated depending the pulsation mode. Figure 11 shows for the model of $1.50 M_{\odot}$ at $\mathrm{Bp}=9 \mathrm{kG}$ the latitudinal variations of radial and horizontal displacements and radiative flux perturbations for the two highest amplitude modes $f_{1}=1372.8 \mu \mathrm{Hz}$ and $f_{2}=1381.5 \mu \mathrm{Hz}$ having $\left(\ell_{\mathrm{m}}, n\right)=(2,21)$ and $\left(\ell_{\mathrm{m}}, n\right)=(1,22)$ identifications, respectively. These include all contributions from $l=0,2,4 \ldots 22(m=0)$ components for $1372.8 \mu \mathrm{Hz}$ mode and all contributions from $\ell=1,3,5 \ldots 23(m=0)$ for the

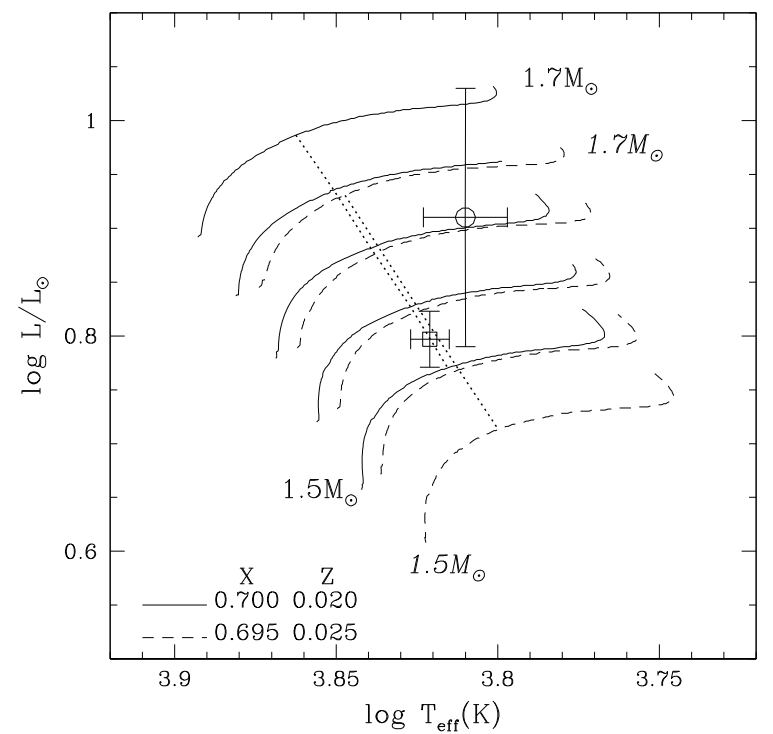

Fig. 7. Evolutionary tracks of stars having masses between $1.5 M_{\odot}$ and $1.7 M_{\odot}$ (with an increment of $0.05 M_{\odot}$ ) and the position of HD 101065 (open square) with error bars. The position from photometric calibration by Kochukhov \& Bangulo (2006) is shown by a circle with error bars. Solid lines are for the chemical composition $(X, Z)=(0.7,0.02)$ and dashed lines for $(X, Z)=(0.695,0.025)$. Dotted lines connect the loci of models whose $\mathrm{p}$-mode frequencies under a strong magnetic field agree best for a given mass with the observed frequencies of HD 101065 .

1381.5 $\mu \mathrm{Hz}$ mode. The aspect changes of disk-integrated pulsation amplitudes, calculated by integrating the latitudinal variations of the radial component of displacement and flux variations over the surface, are shown in Fig. 12. These variations are scaled arbitrarily to maximum amplitude.

As may be seen, the variations in $\cos \alpha$ from 0.55 to 0.36 results in opposite changes in the apparent photometric amplitudes of the two largest amplitude modes - $f_{1}$ tends to increase in an amplitude by about $19 \%$ while $f_{2}$ decreases in amplitude by about $41 \%$. At the same time the apparent RV amplitudes of these modes tends to decrease. The observed weak dependence of the apparent photometric amplitude of the pulsation (which was not resolved into individual modes in the previous analyses of short observing runs) from the polar angle visibility can, in principle, be explained by superposition of different aspect dependencies of apparent amplitudes of individual modes contributing to the observed signal. Among these the opposite changes of the photometric pulsation amplitudes of the $f_{1}$ and $f_{2}$ modes are the dominant contributors. We will not discuss further the rotational variability of the amplitude of pulsations, because there is insufficient observational information about the variability of photometric and radial velocity amplitudes of individual modes. For completeness we mention that variations of the pulsation amplitude and phase over long time intervals is also a common phenomenon for roAp stars (Martinez \& Kurtz 1995) 


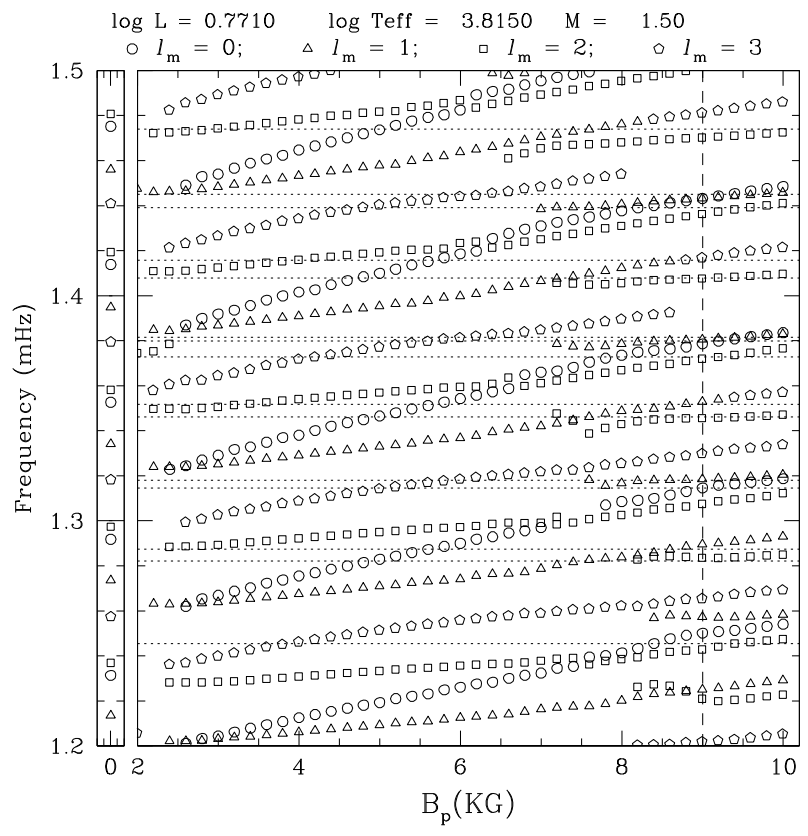

Fig. 8. Frequencies versus the polar strength of magnetic field, $B_{\mathrm{p}}$. Horizontal dashed lines indicate the observed frequencies of HD 101065. This model gives frequencies within $\pm 1.3 \mu \mathrm{Hz}$ range consistent with the observed frequencies around $9 \mathrm{kG}$

Table 4. Best-fit model for each stellar mass.

\begin{tabular}{cccccc}
\hline \hline$M / M_{\odot}$ & $\log T_{\text {eff }}$ & $\log L / L_{\odot}$ & $\operatorname{Age}\left(10^{9} \mathrm{yr}\right)$ & $B_{\mathrm{p}}(\mathrm{kG})$ & $\operatorname{Mean} \operatorname{dev}(\mu \mathrm{Hz})$ \\
\hline \begin{tabular}{c}
$(X, Z)$ \\
\hline
\end{tabular}$(0.70,0.02)$ & & & \\
1.70 & 3.863 & 0.987 & 1.0 & 6.2 & 2.69 \\
1.65 & 3.851 & 0.935 & 1.2 & 7.0 & 2.32 \\
1.60 & 3.840 & 0.882 & 1.3 & 7.6 & 1.36 \\
1.55 & 3.827 & 0.827 & 1.4 & 8.4 & 1.30 \\
1.50 & 3.815 & 0.771 & 1.6 & 9.0 & 1.31 \\
\hline$(X, Z)=(0.695,0.025)$ & & & \\
1.70 & 3.847 & 0.931 & 1.1 & 7.6 & 1.46 \\
1.65 & 3.836 & 0.879 & 1.2 & 8.0 & 1.47 \\
1.60 & 3.824 & 0.823 & 1.3 & 8.2 & 1.49 \\
1.55 & 3.812 & 0.768 & 1.5 & 9.0 & 1.60 \\
1.50 & 3.800 & 0.714 & 1.7 & 9.6 & 1.56 \\
\hline
\end{tabular}

and it cannot be excluded that intrinsic long-term photometric amplitude variation has contributed to the apparent signal.

We note that although our estimate of $B_{\mathrm{p}}=8.7 \mathrm{kG}$ is consistent with the measurements of mean longitudinal field strength spanning $\sim 30$ years, it might be inconsistent (by about a factor 2) with the mean field strength $\langle B\rangle=2.3 \pm 0.4 \mathrm{kG}$ estimated spectroscopically by Cowley et al. (2000) and Shavrina et al. (2006) for HD 101065. A magnetic field strength obtained from a frequency fitting for the roAp star $\gamma$ Equ by Gruberbauer et al. (2008) was also higher than the spectroscopic estimate by Ryabchikova et al. (1997). The discrepancy might arise from a magnetic field that is more complex than simple dipole configuration considered here or from the fact that the mean density of an atmospheric model computed with a standard $T-\tau$ relation is higher than that of Ap stars (Cowley et al. 2000). The atmospheric structure of the star might be significantly modified by the presence of a strong magnetic field. Unperturbed models used in this paper did not include possible magnetic effects
Table 5. Observed and model frequencies for the two best fitting models.

\begin{tabular}{|c|c|c|c|c|}
\hline \multicolumn{5}{|c|}{$\begin{array}{r}M=1.55 M_{\odot}, Z=0.02, \text { Age }=1.4 \times 10^{9} \mathrm{yr}, \text { lo } \\
\log L / L_{\odot}=0.827, B_{\mathrm{p}}=8.4 \mathrm{kG} \\
\text { Mean deviation }=1.303 \mu \mathrm{Hz} .\end{array}$} \\
\hline $\begin{array}{c}\begin{array}{c}\text { Obs. freq. } \\
(\mu \mathrm{Hz})\end{array}\end{array}$ & $\begin{array}{c}\text { Model freq. } \\
(\mu \mathrm{Hz})\end{array}$ & $\bar{l}_{\mathrm{m}}$ & n & $\begin{array}{l}\text { Diff. } \\
(\mu \mathrm{Hz})\end{array}$ \\
\hline 1245.5 & 1244.1 & 2 & 19 & 1.4 \\
\hline 1282.2 & 1284.9 & 2 & 20 & -2.7 \\
\hline 1287.4 & 1289.7 & 1 & 20 & -2.3 \\
\hline 1314.6 & 1314.4 & 0 & 20 & 0.2 \\
\hline 1318.1 & 1319.4 & 1 & 21 & -1.3 \\
\hline 1346.2 & 1346.2 & 2 & 21 & 0.1 \\
\hline 1351.7 & 1353.6 & 1 & 21 & -1.9 \\
\hline 1372.8 & 1372.7 & 2 & 21 & 0.1 \\
\hline 1379.8 & 1379.2 & 0 & 21 & 0.6 \\
\hline 1381.5 & 1381.4 & 1 & 22 & 0.1 \\
\hline 1407.9 & 1408.2 & 2 & 22 & -0.3 \\
\hline 1415.8 & 1417.9 & 3 & 22 & -2.2 \\
\hline 1445.0 & 1443.8 & 1 & 23 & 1.1 \\
\hline 1439.0 & 1437.2 & 2 & 22 & 1.8 \\
\hline 1474.0 & 1470.7 & 2 & 23 & 3.3 \\
\hline \multicolumn{5}{|c|}{$\begin{array}{r}M=1.50 M_{\odot}, Z=0.02, \text { Age }=1.6 \times 10^{9} \mathrm{yr}, \log \\
\log L / L_{\odot}=0.771, B_{\mathrm{p}}=9.0 \mathrm{kG}, \\
\text { Mean deviation }=1.309 \mu \mathrm{Hz}\end{array}$} \\
\hline $\begin{array}{c}\text { Obs. freq. } \\
(\mu \mathrm{Hz})\end{array}$ & $\begin{array}{c}\text { Model freq. } \\
(\mu \mathrm{Hz})\end{array}$ & $\bar{l} l_{\mathrm{m}}$ & n & $\begin{array}{l}\text { Diff. } \\
(\mu \mathrm{Hz})\end{array}$ \\
\hline 1245.5 & 1243.5 & 2 & 19 & 2.0 \\
\hline 1282.2 & 1284.2 & 2 & 20 & -2.0 \\
\hline 1287.4 & 1290.1 & 1 & 20 & -2.7 \\
\hline 1314.6 & 1315.2 & 0 & 20 & -0.6 \\
\hline 1318.1 & 1319.1 & 1 & 21 & -1.0 \\
\hline 1346.2 & 1346.2 & 2 & 21 & -0.01 \\
\hline 1351.7 & 1353.6 & 1 & 21 & -1.9 \\
\hline 1372.8 & 1372.6 & 2 & 21 & 0.2 \\
\hline 1379.8 & 1379.2 & 0 & 21 & 0.6 \\
\hline 1381.5 & 1381.2 & 1 & 22 & 0.2 \\
\hline 1407.9 & 1408.3 & 2 & 22 & -0.4 \\
\hline 1415.8 & 1417.5 & 3 & 22 & -1.8 \\
\hline 1445.0 & 1443.9 & 1 & 23 & 1.1 \\
\hline 1439.0 & 1436.9 & 2 & 22 & 2.1 \\
\hline 1474.0 & 1470.9 & 2 & 23 & 3.0 \\
\hline
\end{tabular}

except for supressing envelope convection and depleting helium in the outermost layers.

\subsection{Radial velocity to light phase shift}

The Johnson $U$ and $B$ photometric and radial velocity amplitudes yielded the velocity-to-amplitude ratio for the principal mode in HD 101065. These ratios are $35.1 \mathrm{~km} \mathrm{~s}^{-1}$ and $45.0 \mathrm{~km} \mathrm{~s}^{-1} \mathrm{mag}^{-1}$ for the $U$ and $B$ band, respectively, and are given also in the fourth column on Table 3 . The corresponding value for the $B$ amplitude is consistent with values found by Leung (1970) for different types of classical pulsating stars $\left(33-76 \mathrm{~km} \mathrm{~s}^{-1} \mathrm{mag}^{-1}\right)$. Note that such a velocity-to-light ratio is based on the averaged amplitude of velocity variations of all spectral lines. It does not have a real physical sense for non-radially pulsating roAp stars without taking into account the surface distributions of chemical elements (Mkrtichian 1994) and the recent discoveries of the short wavelength scale of acoustic oscillations in atmospheres of roAp stars (Baldry et al. 1998; Mkrtichian et al. 2003; 

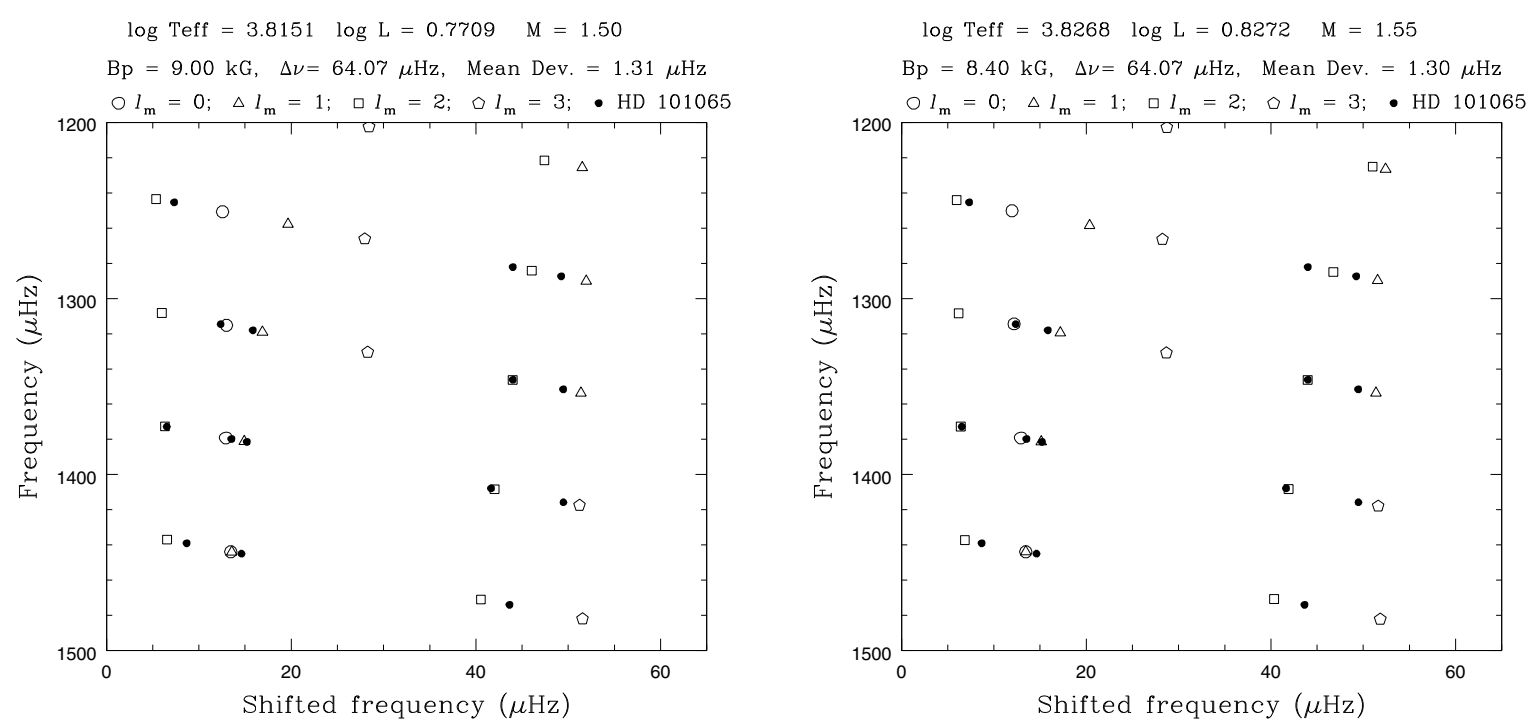

Fig. 9. Echelle-diagram of p-mode oscillation spectrum of HD 101065 (dots) and of the best fit theoretical models with chemical composition $(X, Z)=(0.7,0.02)$ (open symbols). Left: age $=1.4 \times 10^{9} \mathrm{yr}$, mass $=1.55 M_{\odot}, \log T_{\mathrm{e}}=3.8269, \log L / L_{\odot}=0.8272$, and $\mathrm{Bp}=8.4 \mathrm{kG}$. Right: age $=1.6 \times 10^{9} \mathrm{yr}$, mass $=1.50 M_{\odot}, \log T_{\mathrm{e}}=3.8151, \log L / L_{\odot}=0.7709$, and $\mathrm{Bp}=9.0 \mathrm{kG}$.

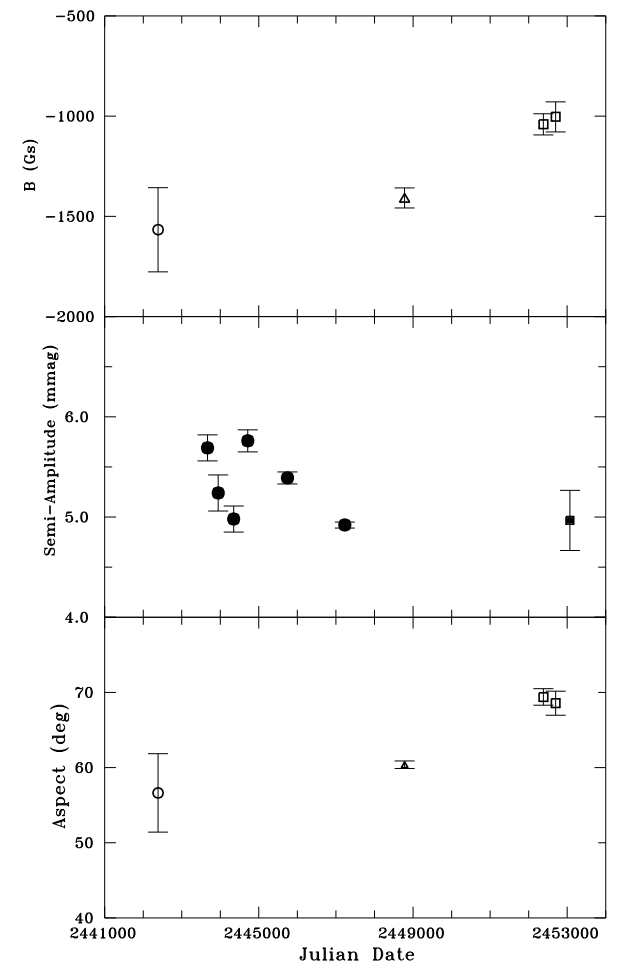

Fig. 10. (Top panel) The magnetic field variability of HD 101065. Open circles - corrected data by Wolff \& Hagen (1976), open triangles - data by Cowley \& Mathys (1998), open squares - by Hubrig et al. (2004). (Middle panel) The long-term $B$-filter semi-amplitude behavior of the principal $f_{1}$ mode. Filled circles - data from Martinez \& Kurtz (1990), filled square - data from our study. (Bottom panel) Magnetic pole aspect change calculated from the magnetic field measurements (top panel) and for a polar field strength $B_{\mathrm{p}}=8.7 \mathrm{kG}$.

Kurtz et al. 2003; Rybchikova et al. 2007b). For HD 101065, as we have already mentioned in Sect. 3 , the pulsation phases of the
Table 6. Mean longitudinal magnetic field measurements for HD 101065 compiled and transformed from Wolff \& Hagen (1976, WH76), Cowley \& Mathys (1998, CM98), and Hubrig et al. (2004, H04). Fourth column lists the expected $\alpha$ for a magnetic pole visibility calculated using a polar magnetic field of $8.7 \mathrm{kG}$ (see text).

\begin{tabular}{|c|c|c|c|c|c|c|}
\hline Year & $\begin{array}{l}\text { JD } \\
2400000+\end{array}$ & $\begin{array}{l}B_{1} \\
\text { Gs }\end{array}$ & $\begin{array}{l} \pm \sigma \\
\text { Gs }\end{array}$ & $\begin{array}{l}\alpha \\
\operatorname{deg}\end{array}$ & $\begin{array}{l} \pm \sigma \\
\operatorname{deg}\end{array}$ & Ref. \\
\hline 1974.92 & 42386.21 & -1567 & 210 & & & \\
\hline 1992.44 & 48782.54 & -1408 & 50 & 60.3 & 0.5 & CM98 \\
\hline 2002.29 & 52383.20 & -1041 & 53 & 69.3 & 1.1 & H04 \\
\hline 2003.17 & 52701.25 & -1004 & 75 & 70.1 & 1.6 & - \\
\hline
\end{tabular}

Table 7. The photometric pulsation semi-amplitude changes for HD 101065 compiled from Martinez \& Kurtz (1990, MK90) and obtained in the present paper (MHS).

\begin{tabular}{lllll}
\hline \hline Year & $\overline{\mathrm{JD}}$ & $\begin{array}{l}A \\
\text { mag }\end{array}$ & $\begin{array}{l} \pm \sigma \\
\text { mag }\end{array}$ & Ref. \\
& $2400000+$ & \\
\hline 1978 & 43666.71 & 5.69 & 0.13 & MK90 \\
1979 & 43945.90 & 5.24 & 0.18 & - \\
1980 & 44344.99 & 4.98 & 0.13 & - \\
1981 & 44713.87 & 5.76 & 0.11 & - \\
1984 & 45743.00 & 5.39 & 0.06 & - \\
1988 & 47228.52 & 4.92 & 0.03 & - \\
2004 & 53067.94 & 4.97 & 0.3 & MHS \\
\hline
\end{tabular}

majority of spectral lines do vary within \pm 0.02 interval (see Fig. 3) and a comparison of averaged radial velocity phase is thus reasonable.

Figure 13 shows the $U$ - and $B$-light curves of HD 101065 along with the radial velocity variations phased with the period of the principal $f_{1}$ mode. The RV and photometry data are binned to 0.05 phase intervals. Zero epoch was taken to be the time of the RV maximum. The bottom panel shows relative radius variations of HD 101065 at the level of the line-forming layers of the atmosphere calculated by integrating the RV curve. Note that the projection factor $p$ (for radial pulsations the projection factor 

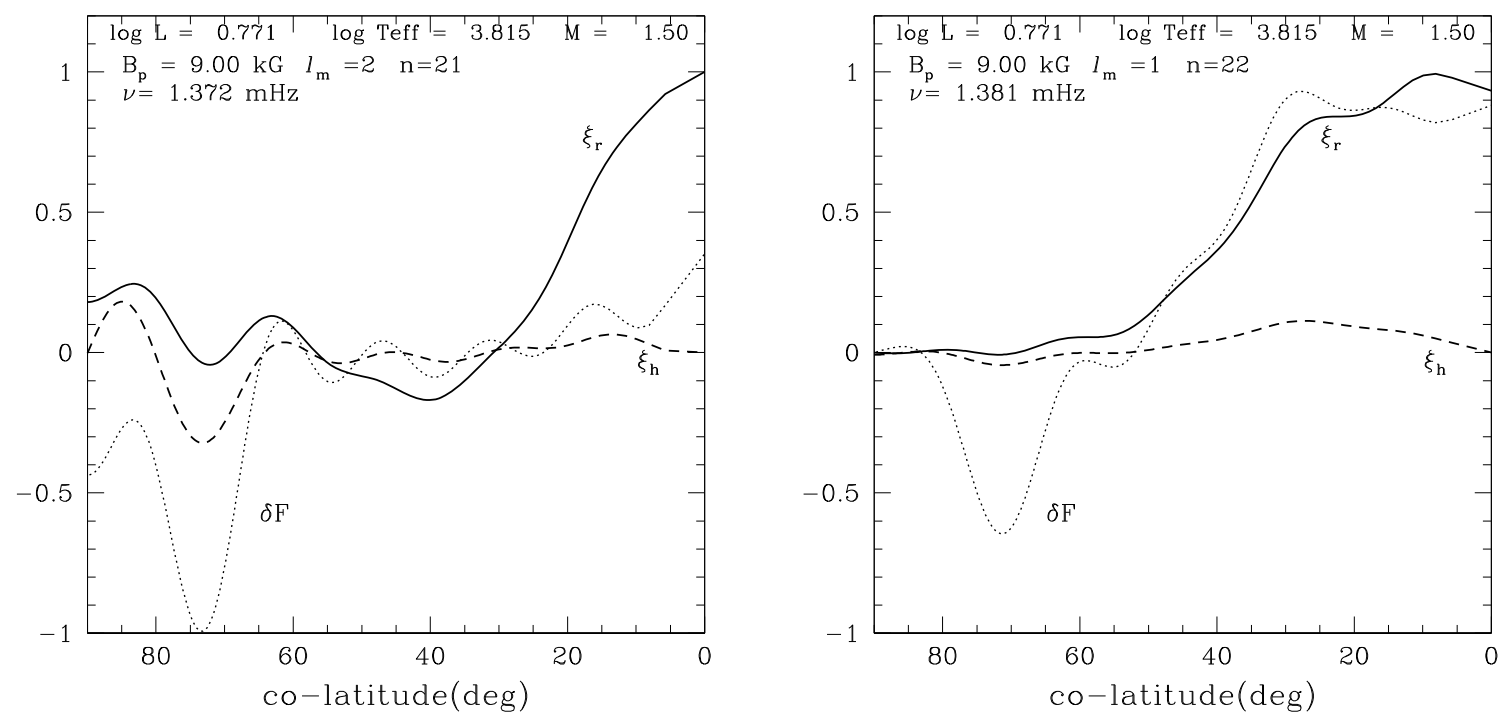

Fig. 11. Latitudinal dependence of the radial (solid line) and horizontal (dashed line) displacements and radiative flux perturbations (dotted lines) for the mode $\left(l_{\mathrm{m}}, n\right)=(2,21)$ (left panel) and $\left(l_{\mathrm{m}}, n\right)=(1,22)$ (right panel).

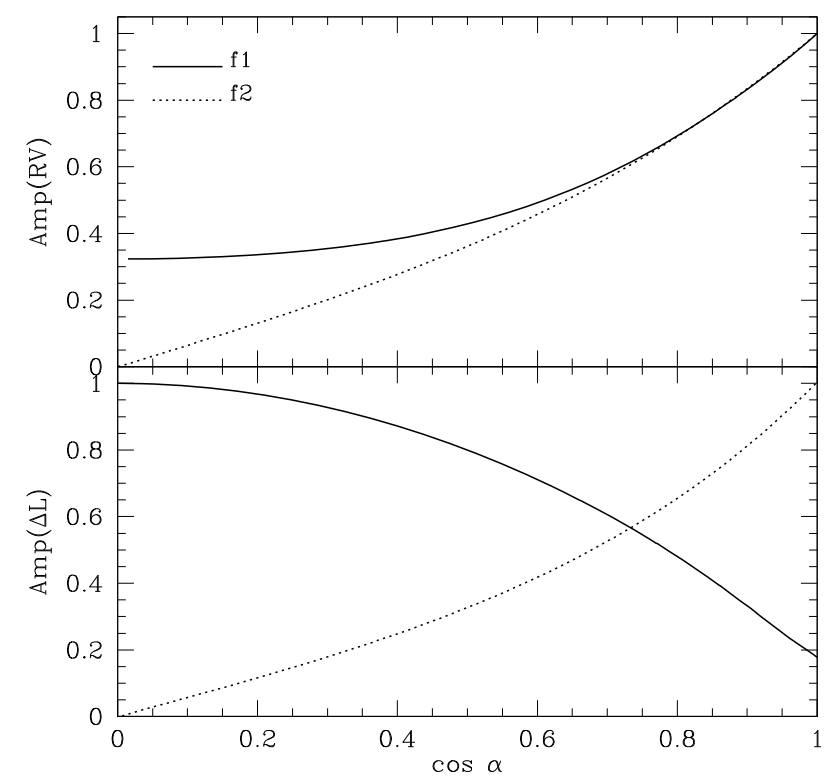

Fig. 12. The disk-averaged pulsation amplitude variability vs. $\cos \alpha$ for $l_{\mathrm{m}}, n=2,21$ and $l_{\mathrm{m}}, n=1,22$ of $\mathrm{HD} 101065$.

is typically $\sim 1.35$ ), that converts the disk-integrated RV amplitude to a surface one was not applied to these relative radius variations.

The remarkable peculiarity seen in Fig. 13 is that light variations are nearly in phase with the RV variations - namely, $\phi\left(R V_{\max }\right)-\phi\left(U_{\max }\right)=0.16$ and $\phi\left(R V_{\max }\right)-\phi\left(B_{\max }\right)=0.19$, respectively. This is contrary to what is seen for classical variables like Cepheids, RR Lyr, or $\delta$ Scuti stars. In these pulsating stars the radial velocity and light curves are mirror images and the maximum of the radial velocity occurs nearly at light minimum (for Cepheids) or between 0.05 to 0.1 phase after the light maximum for $\delta$ Scuti stars (e.g. Smith 1982). The dashed line in Fig. 13 shows the inverse of the observed RV curve which has a "minimum" 0.15-0.19 in phase after the light maximum of the $U$ and $B$ light curves. The inverse RV curve is in better agreement with the expected phase shift between the light and RV curves for classical pulsators. Note that a $0.045 \pm 0.005$ phase lag between the RV maximum and and light minimum (i.e. near coincidence of RV maxima with $\Delta B$ light minima) was found in the roAp star HD 24712 (Matthews et al. 1988). Ryabchikova et al. (2007a) confirmed this result for HD 24712 and found that the brightness maximum occurs for all individual lines after RV maximum, but the phase itself depends strongly on individual lines.

We believe that the differences in the RV-light variations for HD 101065 compared to other pulsating classes of stars and the roAp star HD 24712 in particular, is due to a pulsation phase difference between the spectral line-forming regions of the stellar atmosphere (where the measured RV variations originate) and those layers responsible for the photometric variations. The temperature and displacement node in the roAp star atmospheres might have a different location (Gautschy et al. 1998). Note that the direct link of the amplitudes and phase of $U$ - or $B$-band variations to a geometric depth in the stellar atmosphere is not straightforward for HD 101065 due to the line-rich spectrum and strong line blanketing. Variations in spectral lines can strongly contribute to the photometric variability. On the other hand, the complex co-latitude dependence of brightness and displacement variations shown for two modes $f_{1}$ and $f_{2}$ in Fig. 10 can be additional contributers to phase differences between brightness and RV curves integrated over the disk. Note that correct restoration of acoustic profiles of the atmosphere requires resolution of individual modes of oscillations. These will have different acoustic profiles and different locations of their acoustic nodes in the atmosphere (Mkrtichian et al. 2003). In the present work we resolved 15 oscillation modes. A more detailed multi-mode, lineby-line investigation of acoustic phase and amplitude distribution across the atmosphere of HD 101065 is beyond the scope of the current paper and will be given in a forthcoming paper.

\section{Conclusions}

The analysis of RV data of HD 101065 revealed the rich high-overtone, low degree p-mode oscillation spectrum showing a general spacing between consecutive overtones of modes 


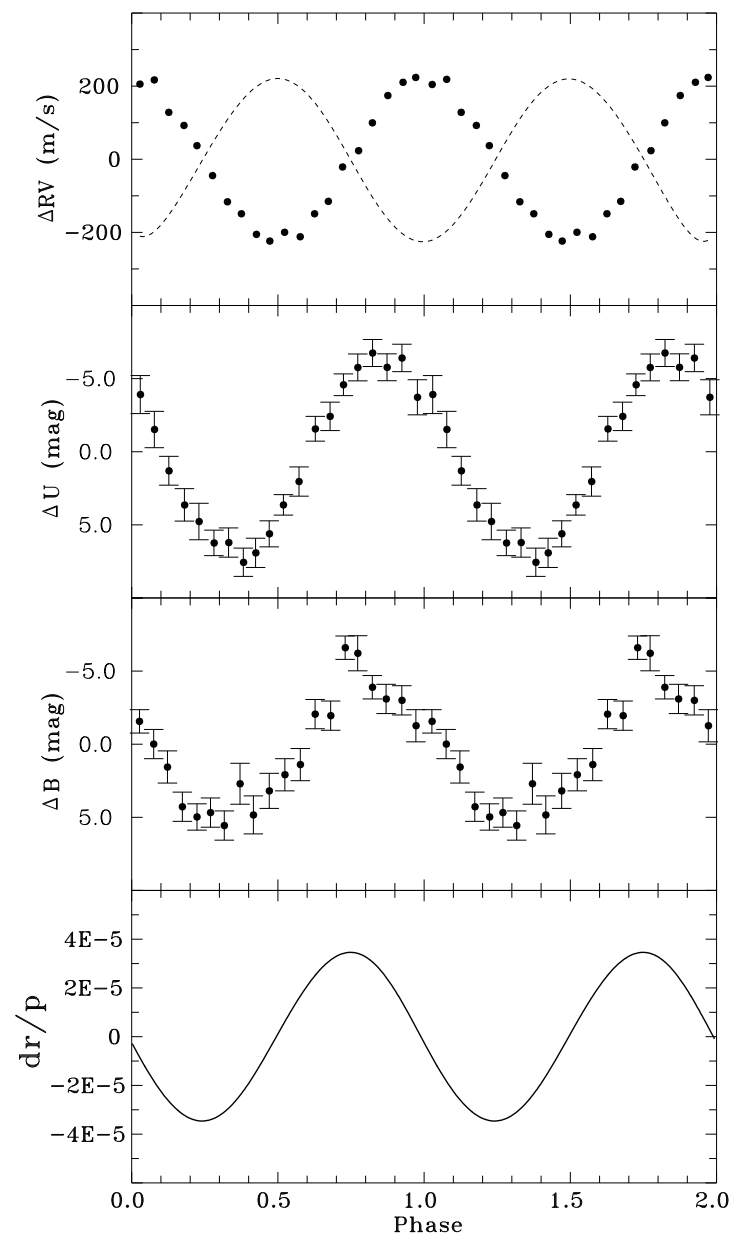

Fig. 13. From top to bottom: the RV, U, B and stellar radius variations phase curves. The accuracy of binned RV averages in the top panel are smaller than the symbol size. The inverted RV curve is shown by the dashed line. The radius variations are not scaled for the projection factor $p$.

$\Delta v=64.07 \pm 0.91 \mu \mathrm{Hz}$ and regular "small" frequency spacings that we interpreted as the result of excitation of the complete spectrum of $\ell=0-2$ modes. From a comparison of observed and calculated nonadiabatic frequencies of axisymmetric modes under different magnetic fields, we found an asteroseismic model for HD 101065. The calculated frequencies agree to within $1.3 \mu \mathrm{Hz}$ of the observed ones. The parameters of the best fit model are: $M=1.525 \pm 0.025 M_{\odot}$, Age $=(1.5 \pm 0.1) \times 10^{9} \mathrm{yr}$, $\log T_{\mathrm{e}}=3.821 \pm 0.006, \log L / L_{\odot}=0.797 \pm 0.026, \log g=$ $4.06 \pm 0.04, \mathrm{Bp}=8.7 \pm 0.3 \mathrm{kG}$. Further progress of stellar parameter determination requires an increase of accuracy of the pulsation frequencies to much better than $1 \mu \mathrm{Hz}$, as well as improvements in pulsation models of magnetic stars. We did the mode identification for all oscillation frequencies. For the dominant mode, the light maximum precedes the time of of RV maximum by phase differences of $\delta \phi_{\mathrm{U}}=0.16(U$-filter $)$ and $\delta \phi_{\mathrm{B}}=0.19(B$ filter). We did not find a long-term correlation between magnetic field and pulsation amplitude variations.

Acknowledgements. The authors thank the anonymous referee for useful comments that improved the quality of the paper. D.E.M. acknowledges his work as a part of research activity of the Astrophysical Research Center for the Structure and Evolution of the Cosmos (ARCSEC) which is supported by the
Korean Science and Engineering Foundation. A.P.H. acknowledges the support of grant 50OW0204 from the Deutsches Zentrum für Luft- und Raumfahrt e.V. (DLR). H.S. thanks Don Kurtz for useful discussions.

\section{References}

Babcock, H. W. 1958, ApJS, 3, 141

Baldry, I. K., Bedding, T. R., Viskum, M., Kjeldsen, H., \& Frandsen, S. 1998, MNRAS, 295, 33

Balmforth, N. J., Cunha, M. S., Dolez, N., Gough, D. O., \& Vauclair, S. 2001, MNRAS, 323, 362

Bidelman, W. P. 2005, in Cosmic Abundances as Records of Stellar Evolution and Nucleosynthesis in honor of David L. Lambert, ed. T. G. Barnes III, \& F. N. Bash, PASPC, 336, 309

Cowley, C. R., \& Mathys, G. 1998, A\&A, 339,165

Cowley, C. R., Ryabchikova, T., Kupka, F., et al. 2000, MNRAS, 317, 299

Cowley, C. R., Bidelman, W. P., Hubrig, S., Mathys, G., \& Bord, D. J. 2004, A\&A, 419, 1087

Cunha, M., \& Gough, D. O. 2000, MNRAS, 319, 1020

Fahlman, G. G., \& Glaspey, J. W. 1973, in Astronomical Observations with Television Type Sensors, ed. J. W. Glaspey, \& G. A. H. Walker (Vancouver, B.V., Institute of Astronomy and Space Science), 347

Fivet, V., Quinet, P., Biémont, É., et al. 2007, MNRAS, 380, 771

Gautschy, A., Saio, H., \& Harzenmoser, H. 1998, MNRAS, 301, 31

Gopka, V. F., Yushchenko, A. V., Shavrina, A. V., et al. 2004, in The A-Star Puzzle, ed. J. Zverko, J. Ziznovsky, S. J. Adelman, \& W. W. Weiss, IAU Symp., 224, 734

Goriely, S. 2007, A\&A, 466, 619

Gruberbauer, M., Saio, H., Huber, D., et al. 2008, A\&A, 480, 223

Hatzes, A. P., \& Mkrtichian, D. E. 2004, MNRAS, 351, 663

Hubrig, S., Kurtz, D. W., Bangulo, S., et al. 2004, A\&A, 415, 661

Iglesias, C. A., \& Rogers, R. J. 1996, ApJ, 464, 943

Kochukhov, O., \& Bagnulo, S. 2006, A\&A, 450, 763

Kurtz, D. W. 1978, IBVS, 1346, 1

Kurtz, D. 1990, ARA\&A, 28, 607

Kurtz, D. W., \& Martinez, P. 2000, Baltic Astron., 9, 253

Kurtz, D. W., Elkin, W. G., \& Mathys, G. 2003, MNRAS, 343, L5

Kurtz, D. W., Elkin, W. G., \& Mathys, G. 2005, MNRAS, 358, L6

Kurtz, D. W., Elkin, W. G., \& Mathys, G. 2006, MNRAS, 370, 1274

Lenz, P., \& Breger, M. 2005, Commun. Asteroseismol., 146, 53

Leung, K. C. 1970, MNRAS, 242, 636

Martinez, P. 1992, PASPC, 30, 371

Martinez, P., \& Kurtz, D. W. 1990, MNRAS, 242, 636

Martinez, P., \& Kurtz, D. W. 1995, PASPC, 83, 58

Matthews, J. M., Wehlau, W. H., Walker, G. A. H., \& Yang, S. 1988, ApJ, 324, 1099

Michaud, G. 1970, ApJ, 160, 641

Mkrtichian, D. E. 1994, Sol. Phys., 152, 275

Mkrtichian, D. E., \& Hatzes, A. P. 2005, A\&A, 26, 185

Mkrtichian, D. E., Hatzes, A. P., \& Kanaan, A. 2003, MNRAS, 345, 781

Palmeri, P., Quinet, P., Biḿont, E., et al. 2007, MNRAS, 374, 63

Piskunov, N. E., Kupka, F., Ryabchikova, T. A., Weiss, W. W., \& Jeffry, C. S. 1995, A\&AS, 112, 525

Przybylski, A. 1961, Nature, 189, 739

Quinet, P., Argante, C., Fivet, V., et al. 2007, A\&A, 474, 307

Romanyuk, I. I. 1984, AISAO, 18, 37

Ryabchikova, T. A., Adelman, S. J., Weiss, W. W., \& Kuschnig, R. 1997, A\&A, 322,234

Rybchikova, T., Sachkov, M., Weiss, W. W., et al. 2007a, A\&A, 462, 1103

Rybchikova, T., Sachkov, M., Kochukhov, O., \& Lyashko, D. 2007b, A\&A, 473, 907

Saio, H. 2005, MNRAS, 360, 1022

Saio, H., \& Gautschy, A. 2004, MNRAS, 350, 485

Shibahashi, H., \& Saio, H. 1985, PASJ, 37, 245

Shavrina, A. V., Polosukhina, N. S., Pavlenko, Ya. V., et al. 2003, A\&A, 409, 707

Shavrina, A. V., Polosuhina, N. S., Han, S., et al. 2006, ARep, 50, 500

Smith, M. A. 1982, ApJ, 254, 242

Valenti, J. A., Butler, R. P., \& Marcy, G. W. 1995, PASP, 107, 966

Wegner, G., \& Petford, A. D. 1974, MNRAS, 168, 557

Wolff, S. C., \& Hagen, W. 1976, PASP, 88, 119

Yushchenko, A., Gopka, V., Goriely, S., et al. 2006, Proceedings of the International Symposium on Nuclear Astrophysics - Nuclei in the Cosmos - IX, 25-30 June 2006, CERN, 198 